\title{
Interactive comment on "Remote monitoring of seismic swarms and the August 2016 seismic crisis of Brava, Cape Verde, using array methods" by Carola Leva et al.
}

\section{Carola Leva et al.}

leva@geophysik.uni-frankfurt.de

Received and published: 22 October 2020

Response to the interactive comment on "Remote monitoring of seismic swarms and the August 2016 seismic crisis of Brava, Cape Verde, using array methods" by Carola Leva et al.

Reviewer 2 - Carmen López

We would like to thank the reviewer for the helpful comments and constructive suggestions.

Printer-friendly version

Discussion paper

R2: I find the paper by Leva et al. (2020) of great interest, since it shows how precur- 
sory volcanic activity behaves in oceanic islands; there are not many scientific papers of this type. In oceanic islands, volcanic activity monitoring involves great difficulty due to out-of-network seismic occurrence and poor network coverage, which does not facilitate the full study of the precursor phenomena. Tracking the seismic activity that accompanies the unrest is truly challenging, thus I find this paper of interest. I will now provide some recommendations and comments that I hope will be useful. Authors propose an intelligent approach, which is increasingly used in oceanic islands and submarine volcanism, the use of seismometer arrays, which by decreasing the signal-to-noise ratio can detect low amplitude signals, even below the ambient noise. These arrays are optimal for detection, but not so good for localization, giving notable errors in azimuth and distance, especially in the case of no calibrated array and also in the case of using plane wave front approximation instead of a spherical one. Sections describing the methodology are well developed with a careful application to data and errors estimation. Array analysis was performed in the time domain, being able to locate volcano tectonic (VT) events. I wonder, if an additional analysis in the frequency domain ( $\mathrm{F}-\mathrm{K}$ analysis) had been carried out, whether it would have also characterized low frequency tremor or LP signals, which have not been included in the study. In fact (line 29) according to data recorded by a permanent seismic monitoring network (Faria and Day, 2017), the crisis comprised about 1000 shallow earthquakes and tremors.

A.: We thank the reviewer for the appreciation of our work. The tremor signals produce smaller amplitudes, which are likely suppressed by noise in the distance of the array of $35 \mathrm{~km}$. We did not carry out a F-K analysis, but could not find any indication for such signals by manual inspection of the seismograms using different filters. Additionally, we applied different sta-/lta-triggers to detect events of different frequency content and could not find any tremors or long-period events that originated on Brava, especially during the seismic crisis in August 2016.

R2: The localized events set their depth at $5 \mathrm{~km}$, without assessing the error associated with this setting. I think other depths should be tested to know its impact on location.

Printer-friendly version

Discussion paper 
A.: We performed a careful analysis of the contributions to the error of the epicentral distance by evaluating the influence of all parameters used for the distance estimation. It turned out, that a variation of the event depth only has a minor impact on the result, compared to other variables (lines 91, 94-97). After this error analysis we found that an error of $10 \%$ for the distance in general covers best the errors resulting from the uncertainties of the distance estimation (lines 97/98). We decided to use this relative conservative estimate for the error to incorporate the uncertainties of the simple twolayer assumption, including the uncertainties of the depth. This is already described in the text, but we will clarify this point during revision.

R2: I think it would be desirable to get additional data, mainly about gas emissions and surface deformations, or additional seismic information for the better identification of the different stages. A.: Yes, we agree that it would be desirable to have additional data. To our knowledge, there are other groups working on a publication about gas emission data. Unfortunately, the data is not available to us. The seismic data of the local monitoring network is also restricted.

R2: At this regard, it would be useful to include in Figure 3 the accumulated number of events.

A.: Thank you for the suggestion, we will modify the Figure accordingly.

R2: The variations of the "b" parameter should be discussed in more detail. During eruptive unrest phenomena, in other volcanic islands, strong variations of the "b" parameter have been observed, from values greater than 2, to close to 1, and in all cases reflecting precursory dynamic activity with swarms of VT-type events. It would also be necessary to add a figure with the temporal evolution of the "b" value.

A.: Thank you for pointing this out. It is difficult to assess the precise b-value as we deal with rather low numbers of events (line 129). However, we will clarify how we estimate the $b$-value and add a figure of the $b$-value for the complete study period. We have also looked at the b-value variation within 3 month intervals and will add the corresponding

Printer-friendly version Discussion paper 
figures to the supplementary material. For a more detailed interpretation we would need longer observation times of several years.

R2: Figures show that seismicity fluctuates almost constantly, and only in certain periods is concentrated in-land, always showing dispersion. It is very possible that the dispersion is partly a product of the limitation of the array, in fact, a radial distribution of the epicenters with centre in the array is observed, showing that the semi-major axis of the error coincides with the geometry of the event cloud (fig. 6b). In this regard, if possible, it would be desirable to include the error ellipses in all locating figures (Figure 5 a, b,; Figure 6a, Figure 8 a, b, Figure 9).

A.: This is only apparently the case, in other months this is not observed. Possibly this apparent dispersion could, under consideration of the error of the backazimuth, be interpreted as an indicator, that the events cluster more closely. Nevertheless, we observe a relative shift of the event locations over the study period. A detailed analysis shows that there is a systematic difference in events west and south of Brava, which cannot be explained by a random error in BAZ. We decided to not include the error ellipses in all figures, as this strongly influences the readability of the maps. Nevertheless, we will add a figure with the error ellipses to the supplementary material.

R2: The authors state that they do not observe tremor or LP signals, but the array technique used (beamforming in time) is not the best for these type of low frequency events, so I think their existence cannot be ruled out, please it can be included a clarification.

A.: Please refer to our response to an earlier question above. We do not rule out the existence of different event types that could be a precursor of the crisis, we suggest that their absence in our data can be explained by the rather large distance of $35 \mathrm{~km}$ between array and possible source locations near Brava (line 166). We do not observe other event types originating from Brava. From the earthquake analysis we also do not find precursors. However, we will clarify this in the revised manuscript.

Printer-friendly version

Discussion paper 
R2: I believe a further discussion about the interpretation of the phenomena is needed. The authors state "We conclude that the seismic crisis might be an example of a failed eruption, likely caused by the transport of magma and / or CO2 into the upper crust, as it has been suggested by the observed changes on diffuse $\mathrm{CO} 2$ degassing surveys ", lines 230-232. To state that, it would be necessary to analyse results with data from local monitoring networks, including gas emission and, if it was the case, deformation, occurring during the studied period. In addition, an interpretation based on the knowledge of the structure and the geological frame would be recommended.

A.: Yes, we agree that it would be interesting to directly compare the gas emission data and the data of the local monitoring network to our data. Unfortunately, they are not available to us. In the revised manuscript we have included a discussion about a possible uplift period in 2016. However, more data, especially of a possible deformation, would be desirable. We will include an outlook in the conclusions, pointing out the necessity of including information from other disciplines to better assess volcanic hazards.

Additional comments taken from the annotated manuscript

Line 90: I do not understand why all events set their depth at $5 \mathrm{~km}$. I think other depths should be tested to know its impact, and select which one minimize errors.

A.: We tested the impact of different depths (and different crustal and mantle velocities as well as different Moho depths). We understand, that our description of this error analysis (line 94-100) might be misleading and we will clarify this point during revision.

Line 112: In my understanding, the periods referred to from here on, are not presented month by month. And I have some difficulties in understand the reasons for time periods selection. Please clarify the distinctive characteristics of each one of them.

A.: Thank you for pointing this out. We described the periods with elevated seismicity for each month. We will clarify this in the revised manuscript.

Printer-friendly version

Discussion paper 
Line 136: Why activity in this period is not considered as a seismic crisis? between 29-30 November you have even more events that in previous periods.

A.: The term seismic crisis referred to the period with elevated seismicity beneath Brava, leading to evacuation of a village on Brava. The increased activity from 29 November to 2 December occurred offshore and the alert level for Brava was not raised.

Interactive comment on Nat. Hazards Earth Syst. Sci. Discuss., https://doi.org/10.5194/nhess2020-225, 2020. 